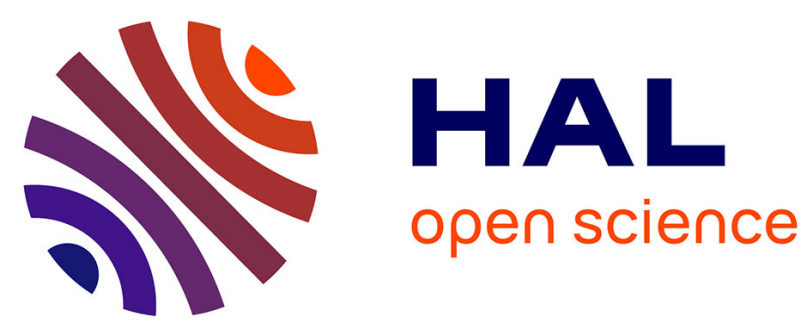

\title{
Propagation, Diffusion and Randomization in Cellular Automata
}

Guillaume Theyssier

\section{To cite this version:}

Guillaume Theyssier. Propagation, Diffusion and Randomization in Cellular Automata. 22th International Workshop on Cellular Automata and Discrete Complex Systems (AUTOMATA), Jun 2016, Zurich, Switzerland. pp.3-9, 10.1007/978-3-319-39300-1_1 . hal-01435029

\section{HAL Id: hal-01435029 \\ https://hal.inria.fr/hal-01435029}

Submitted on 13 Jan 2017

HAL is a multi-disciplinary open access archive for the deposit and dissemination of scientific research documents, whether they are published or not. The documents may come from teaching and research institutions in France or abroad, or from public or private research centers.
L'archive ouverte pluridisciplinaire HAL, est destinée au dépôt et à la diffusion de documents scientifiques de niveau recherche, publiés ou non, émanant des établissements d'enseignement et de recherche français ou étrangers, des laboratoires publics ou privés. 


\title{
Propagation, diffusion and randomization in cellular automata
}

\author{
Guillaume Theyssier \\ Insitut de Mathématiques de Marseille (CNRS, AMU, Centrale Marseille)
}

\begin{abstract}
A large part of the study of of cellular automata dynamics consists in comparing pairs of configurations and their orbits. Here we focus on pairs of configurations having a finite number of differences (diamonds) like in the celebrated Garden-of-Eden theorem. First, we show that it allows to study expansive-like phenomena where classical notions of chaotic behavior like positive expansivity or strong transitivity don't apply (in reversible CAs for instance). Second, we establish that for a large class of linear CA, diffusion of diamonds is equivalent to randomization (a large class of probability measures converge weakly towards the uniform measure under the action of the CA). Both properties are also equivalent to the absence of gliders in the CA. Finally, we give examples of reversible linear CAs that are strong randomizers (the convergence towards the uniform measure is simple and not only in Cesaro mean). This strong behavior is however provably impossible with linear CA having commuting coefficients (e.g. linear CA over cyclic groups).
\end{abstract}

Forewords This extended abstract is based upon unpublished works in collaboration with A. Gajardo and V. Nesme on one hand (pre-expansivity), and B. Hellouin de Menibus and V. Salo on the other hand (randomization).

\section{Introduction}

Cellular automata are dynamical systems and standard definitions and tools from the general theory can be considered to study them $[3,4,14,2]$. Concerning topological dynamics for instance, the classical notion of (positive) expansivity has been applied to CA giving both a rich theory in the one-dimensional case $[1,4,12]$ and a general inexistence result in essentially any other setting $[17,13]$. Besides, in the ergodic dynamics settings, a lot of work was accomplished to show examples of randomizing behaviors (sometimes depicted as a kind of the second law of thermodynamics): a large class of probability measures (weakly) converge towards the uniform measure under the action of the CA $[5,9,15,16,6]$ (see [14] for a general review of ergodic theory of CA).

Cellular automata also have a particular structure and properties that don't necessarily admit sensible formalization in the general setting of topological/ergodic dynamics. For instance, the space of configurations allows to define the notion of diamonds: two configurations that differ only on finitely many positions of 
the lattice. The Garden of Eden theorem, which has a long history [3,10,11,7,2] and is emblematic of this CA specific theoretical development, then says that surjectivity is equivalent to pre-injectivity (injectivity on diamonds) if and only if the lattice is given by an amenable group.

In this extended abstract we introduce basic definitions about possible evolutions of diamonds under the action of a CA: gliders, pre-expansivity, diffusion. We then show that this point of view allows to characterize (weak) randomization combinatorially and link it to pre-expansivity in a large class of linear cellular automata. We also give reversible examples with interesting behaviors in this context: pre-expansivity without positive expansivity and strong randomization.

\section{Formal definitions}

We consider spaces of configurations of the form $Q^{\mathbb{Z}^{N}}$ for some $N \geq 1$ and $Q$ a finite set. Given two configurations $c$ and $d$, we denote by $\Delta(c, d)$ the set of positions where they differ:

$$
\Delta(c, d)=\left\{z \in \mathbb{Z}^{N}: c(z) \neq d(z)\right\} .
$$

Two configurations $c, d$ are asymptotic, denoted $c \stackrel{\infty}{=} d$, if they differ only in finitely many positions: $\Delta(c, d)$ is finite. When $c \stackrel{\infty}{=} d$ and $c \neq d$, we say it is a diamond and denote it by $c \diamond d$.

A cellular automaton $F$ is an endomorphism of $Q^{\mathbb{Z}^{N}}$ characterized by a local transition map $f: Q^{V} \rightarrow Q$, where $V \subset \mathbb{Z}^{N}$ is finite (called neighborhood of $F$ ), and defined as follows:

$$
\forall c \in Q^{\mathbb{Z}^{N}}, \forall z \in \mathbb{Z}^{N}, F(c)(z)=f\left(\left.v \in V \mapsto c\right|_{z+v}\right)
$$

\subsection{Diamonds and their dynamics}

We are interested in the evolution of diamonds under the evolution of a given CA. More precisely, we look at the sequence:

$$
\Delta_{F}^{t}(c, d)=\Delta\left(F^{t}(c), F^{t}(d)\right)
$$

when $t$ grows and $c \diamond d$.

If $E \subseteq \mathbb{Z}^{N}$ is finite, we denote by $\operatorname{diam}(E)$ its diameter, i.e. the largest distance (Manhattan) between any two of its elements.

Definition 1 (Glider). $A$ glider for a $C A F$ is a diamond $c \diamond d$ such that there is a bound $B$ with $\operatorname{diam}\left(\Delta_{F}^{t}(c, d)\right) \leq B$. for all $t$.

By the Garden-of-Eden (or Moore-Myhill) Theorem, any non-surjective CA admits a glider: it's not pre-injective so it admits a diamond $c \diamond d$ such that $\Delta_{F}^{t}(c, d)=\emptyset$ for all $t \geq 1$. The converse is false (some surjective CA also admit gliders). In the following we are essentially interested in surjective CA. 
Definition 2 (Pre-expansivity). $F$ is pre-expansive if there exists a finite observation window $W \subseteq \mathbb{Z}^{N}$ such that, for any diamond $c \diamond d$, there is some time $t \geq 0$ such that $\Delta_{F}^{t}(c, d) \cap W \neq \emptyset$.

Given a set of integers $X \subseteq \mathbb{N}$ we denote by $d_{+}(X)$ its (upper) density:

$$
d_{+}(X)=\limsup _{n \rightarrow \infty} \frac{\# E \cap\{1, \ldots, n\}}{n} .
$$

When considering a sequence $\left(s_{n}\right)$, its density is the density of its support.

Definition 3 (Diffusivity). $F$ is diffusive if for any diamond $c \diamond d$ we have

$$
\# \Delta_{F}^{t_{n}}(c, d) \underset{n \rightarrow \infty}{\longrightarrow} \infty
$$

on an increasing sequence $\left(t_{n}\right)$ of density one. $F$ is strongly diffusive if the above limit holds without taking a subsequence of $\mathbb{N}$, i.e. if

$$
\# \Delta_{F}^{t}(c, d) \underset{t \rightarrow \infty}{\longrightarrow} \infty
$$

We will sometimes speak about weak diffusivity to emphasize that it is not strong diffusivity.

\section{$2.2 \quad$ Ergodic dynamics}

A (finite) pattern is a partial configuration of finite support: $u: \operatorname{Dom}(u) \subseteq \mathbb{Z}^{N} \rightarrow Q$ with $\operatorname{Dom}(u)$ finite. The cylinder $[u]$ associated to such a pattern $u$ is the set of configuration:

$$
[u]=\left\{c \in Q^{\mathbb{Z}^{N}}: \forall z \in \operatorname{Dom}(u), u(z)=c(z)\right\}
$$

A Borel probability measure over $Q^{\mathbb{Z}^{N}}$ is completely determined by a map $\mu$ from cylinders to $[0 ; 1]$ such that:

1. $\mu\left(Q^{\mathbb{Z}^{N}}\right)=1$

2. if $[u]=\bigcup_{k}\left[u_{k}\right]$ with $\left[u_{k}\right]$ pairwise disjoint then $\mu([u])=\sum_{k} \mu\left(\left[u_{k}\right]\right)$

A measure is shift-invariant if $\mu(E)=\mu\left(\sigma_{z}(E)\right)$ for any (measurable) set $E$ and any translation $z \in \mathbb{Z}^{N}$.

A Bernoulli measure is a shift invariant product measure in the sense that the measure of any cylinder $[u]$ is given by the product:

$$
\mu([u])=\prod_{z \in \operatorname{Dom}(u)} \mu^{*}(u(z))
$$

where $\left(\mu^{*}(q)\right)_{q \in Q}$ is the probability vector on letters of the alphabet that characterizes $\mu$. It is non-degenerate if $0<\mu^{*}(q)<1$ for all $q \in Q$. An important 
particular case is the uniform measure $\mu_{0}$ : the Bernoulli measure where all $\mu^{*}(q)$ are equal to $\frac{1}{\# Q}$. by:

Given a $\mathrm{CA}^{2} F$ and a measure $\mu$, the image measure $F \mu$ is the measure given

$$
F \mu([u])=\mu\left(F^{-1}([u])\right) .
$$

All surjective CA preserve the uniform measure $\left(F \mu_{0}=\mu_{0}\right)$ and conversely any $\mathrm{CA}$ that preserves the uniform measure is surjective $[3,8]$.

Definition 4 (Randomization). Given a class of measures $X$, we say that $F$ is randomizing if for any $\mu \in X$ and any cylinder $[u]$

$$
\lim _{n \rightarrow \infty} F^{t_{n}} \mu([u])=\mu_{0}([u])=(\# Q)^{-\# \operatorname{Dom}(u)}
$$

on an increasing sequence $\left(t_{n}\right)$ of density 1.

$F$ is strongly randomizing if the above limit holds without taking a subsequence of $\mathbb{N}$, i.e. if

$$
\lim _{t \rightarrow \infty} F^{t} \mu([u])=\mu_{0}([u])=(\# Q)^{-\# \operatorname{Dom}(u)} .
$$

This definition depends of course on the choice of $X$. In the sequel and without any explicit mention, $X$ will always be the set of non-degenerate Bernoulli measures.

\section{The ideal world of 1D Abelian linear cellular automata}

This section aims at developing a rather complete analysis of diamond dynamics under two strong assumptions:

- in 1D, any incoming information arrives either from the left or from the right, and must cross any large enough window;

- for linear CA, it is sufficient to study finite configurations (i.e. almost everywhere equal to state 0 ) to actually understand all possible diamonds.

Definition 5. Let $(Q, \oplus)$ be a finite Abelian group and denote by $\bar{\oplus}$ the componentwise extension of $\oplus$ to $Q^{\mathbb{Z}^{N}}$. A CAF over $Q^{\mathbb{Z}^{N}}$ is linear for $(Q, \oplus)$ if

$$
\forall c, d \in Q^{\mathbb{Z}^{N}}: F(c \bar{\oplus} d)=F(c) \bar{\oplus} F(d)
$$

Equivalently, such a $C A$ can be written as:

$$
F(c)_{z}=\sum_{i \in V} h_{i}\left(x_{z+i}\right)
$$

where $V \subseteq \mathbb{Z}^{N}$ is finite and the $h_{i}$ are homomorphisms of the group $(Q, \oplus)$. A $C A$ which is linear for some Abelian group is called Abelian CA in the following.

The following lemma justifies definitions of previous section, in particular the interest of restricting to subsequences of time steps of density 1.

Lemma 1. An Abelian CA is diffusive if and only if it has no glider. In particular, a pre-expansive Abelian CA is always diffusive. 


\subsection{A characterization of randomizing CA}

A character of a group $\mathcal{G}$ is a continuous group homomorphism $\mathcal{G} \rightarrow \mathbb{T}^{1}$, where $\mathbb{T}^{1}$ is the unit circle group (under multiplication). Denote $\mathcal{\mathcal { G }}$ the group of characters of $\mathcal{G}$. Given an Abelian group $(Q, \oplus)$, the set of configurations $Q^{\mathbb{Z}^{N}}$ forms an Abelian group by component-wise application of $\oplus$. When considering a linear CA $F$ for the group $(Q, \oplus)$ and a character $\chi \in Q^{\mathbb{Z}^{N}}$, then $\chi \circ F$ is also a character because $F$ is an homomorphism of $Q^{\mathbb{Z}^{N}}$.

$Q^{\mathbb{Z}^{N}}$ is in bijective correspondence with the sequences of $(\hat{Q})^{\mathbb{Z}^{N}}$ whose elements are all 1 (constant map equal to 1 ) except for a finite number. That is, $\chi \in Q^{\hat{Z}^{N}}$ can be written as $\chi(x)=\prod_{k \in \mathbb{Z}^{N}} \chi_{k}\left(x_{k}\right)$ where all but finitely many elements $\chi_{k}$ are equal to 1 . For a given $\chi$, the number of nontrivial $\chi_{k}$ in the sequence is its rank denoted $\operatorname{rank}(\chi)$.

The Fourier coefficient of a measure $\mu$ associated to a character $\chi$ is:

$$
\hat{\mu}[\chi]=\int_{Q^{Z^{N}}} \chi d \mu=\sum_{u \in Q^{D}} \mu([u]) \prod_{k \in D} \chi_{k}\left(u_{k}\right)
$$

where $D$ is any finite set that contains all $\chi_{k}$ which are not 1 . We this definition we have

$$
(\hat{F \mu})[\chi]=\hat{\mu}[\chi \circ F] .
$$

Fourier coefficients characterize measures in the following sense:

$$
\forall u, \mu_{n}([u]) \rightarrow \mu_{\infty}([u]) \text { if and only if } \forall \chi, \hat{\mu}_{n}[\chi] \rightarrow \hat{\mu}_{\infty}[\chi] .
$$

In $[15,16]$, the class of harmonically mixing measures is considered: $\mu$ is harmonically mixing if its Fourier coefficients uniformly go to zero when the rank of characters increase, formally: for all $\varepsilon>0$, there exists a $R>0$ such that $\operatorname{rank}(\chi)>R \Rightarrow \hat{\mu}[\chi]<\varepsilon$. This class contains many natural measures, in particular any non-degenerate Bernoulli and Markov measure $[15,16]$. The interest of the definition is that if one wants to show that a linear CA $F$ (strongly) randomizes all harmonically mixing measures, then it is sufficient to show that

$$
\operatorname{rank}\left(\chi \circ F^{t}\right) \rightarrow_{t} \infty
$$

for all character $\chi$ (or that the limit holds on a subsequence of time steps of density 1 for the case of weak randomization).

This property (weak version) was introduced in $[15,16]$ using the adjective "diffusive". To avoid confusion, we will use the term "chi-diffusive" here.

The following Theorem gives a characterization of randomization for a large class of Abelian CA. It shows in particular that the sufficient condition of chidiffusivity introduced is actually necessary as soon as we ask for randomization of a class of measures that contain non-degenerate Bernoulli measures. The key idea behind the theorem is that composing a character with iterations of a linear CA $F$ over $Q^{\mathbb{Z}^{N}}$ can be seen as the action of a new cellular automaton $\hat{F}$ (its dual) acting on the space $(\hat{Q})^{\mathbb{Z}^{N}}$. It is then possible to show that $F$ and $\hat{F}$ are close enough dynamically so that they share properties like diffusion. 
Theorem 1. Let $F$ be a linear CA over the group $\mathbb{Z}_{p^{l}}^{k}$ for some prime $p$ and $l, k \geq 1$. Then the following are equivalent:

- $F$ has no glider;

- $F$ is diffusive;

- $F$ is chi-diffusive;

- F randomizes any non-degenerate Bernoulli measure;

- F randomizes any harmonically mixing measure.

Corollary 1. For linear $C A F, G$ verifying the hypotheses of the previous theorem we have:

- if $F$ is pre-expansive then $F$ randomizes all harmonically mixing measures;

- $F$ and $G$ randomize harmonically mixing measures if and only if $F \times G$ does.

\subsection{Interesting reversible examples}

In this subsection, we consider examples of Abelian CA over groups of the form $Q=\mathbb{Z}_{p}^{2}$. To facilitate notations, we represents elements of $Q$ as vectors and use matrix notation to represent homomorphisms of $Q$.

Proposition 1. Let $F_{3}$ be the linear $C A$ over $\mathbb{Z}_{3}^{2}$ defined by:

$$
F_{3}(c)_{z}=\left(\begin{array}{ll}
1 & 0 \\
0 & 0
\end{array}\right) \cdot c_{z-1}+\left(\begin{array}{ll}
1 & 1 \\
1 & 0
\end{array}\right) \cdot c_{z}+\left(\begin{array}{ll}
1 & 0 \\
0 & 0
\end{array}\right) \cdot c_{z+1}
$$

where the addition is done in $\mathbb{Z}_{3}^{2} . F_{3}$ is pre-expansive but not positively expansive because reversible.

Proposition 2. Let $G_{2}$ be the linear $C A$ over $\mathbb{Z}_{2}^{2}$ defined by:

$$
G_{2}(c)_{z}=\left(\begin{array}{ll}
1 & 1 \\
1 & 0
\end{array}\right) \cdot c_{z}+\left(\begin{array}{ll}
1 & 0 \\
0 & 0
\end{array}\right) \cdot c_{z+1}
$$

where the addition is done in $\mathbb{Z}_{2}^{2} . G_{2}$ is strongly randomizing.

We don't have any characterization of strong randomization. However, there is a natural class of linear CA where strong randomization is impossible whereas weak randomization can happen.

Definition 6 (super-Abelian CA). A linear CA F over an Abelian group of the form

$$
F(c)_{z}=\sum_{i \in V} h_{i}\left(x_{z+i}\right)
$$

is super-Abelian if the homomorphisms $h_{i}$ commute pairwise.

The class of super-Abelian CA includes all linear CA over cyclic groups, but also all linear CA whose coefficients are just scalars (called LCA in $[15,16,6]$ ).

The intuition in the following proposition is that commutation in a superAbelian CA induces too many cancellations of coefficients at specific time steps, so that infinitely many iterations go back close to the initial (non-uniform) measure.

Proposition 3. No super-Abelian CA is strongly randomizing. 


\section{References}

1. François Blanchard and Alejandro Maass. Dynamical properties of expansive onesided cellular automata. Israel Journal of Mathematics, 99:149-174, 1997.

2. Tullio Ceccherini-Silberstein and Michel Coornaert. Cellular Automata and Groups. Springer, 2010.

3. Gustav Arnold Hedlund. Endomorphisms and automorphisms of the shift dynamical system. Mathematical Systems Theory, 3:320-375, 1969.

4. Petr Kůrka. Topological and symbolic dynamics. Société Mathématique de France, 2003.

5. D.A. Lind. Applications of ergodic theory and sofic systems to cellular automata. Physica D: Nonlinear Phenomena, 10(1-2):36 - 44, 1984.

6. Alejandro Maass, Servet Martínez, Marcus Pivato, and Reem Yassawi. Asymptotic randomization of subgroup shifts by linear cellular automata. Ergodic Theory and Dynamical Systems, 26:1203-1224, 82006.

7. Antonio Machi and Filippo Mignosi. Garden of eden configurations for cellular automata on Cayley graphs of groups. SIAM journal on discrete mathematics, 6(1):44, February 1993.

8. A. Maruoka and M. Kimura. Condition for Injectivity of Global Maps for Tessellation Automata. Information and Control, 32:158-162, 1976.

9. Munemi Miyamoto. An equilibrium state for a one-dimensional life game. J. Math. Kyoto Univ., 19(3):525-540, 1979.

10. Edward F. Moore. Machine models of self-reproduction. In A. W. Burks, editor, Essays on Cellular Automata, pages 187-203. University of Illinois Press, 1970.

11. John Myhill. The Converse of Moore's Garden-of-Eden Theorem. In Proceedings of the American Mathematical Society, volume 14, pages 658-686. American Mathematical Society, 1963.

12. Masakazu Nasu. Nondegenerate q-biresolving textile systems and expansive cellular automata of onesided full shifts. Transactions of the American Mathematical Society, 358:871-891, 2006.

13. Marcus Pivato. Positive expansiveness versus network dimension in symbolic dynamical systems. Theor. Comput. Sci., 412(30):3838-3855, 2011.

14. Marcus Pivato. The ergodic theory of cellular automata. Int. J. General Systems, 41(6):583-594, 2012.

15. Marcus Pivato and Reem Yassawi. Limit measures for affine cellular automata. Ergodic Theory and Dynamical Systems, 22:1269-1287, 82002.

16. Marcus Pivato and Reem Yassawi. Limit measures for affine cellular automata ii. Ergodic Theory and Dynamical Systems, 24:1961-1980, 122004.

17. Mark A. Shereshevsky. Expansiveness, entropy and polynomial growth for groups acting on subshifts by automorphisms. Indagationes Mathematicae, 4(2):203 - 210, 1993. 